\title{
Magnetism, superconductivity, and pairing symmetry in Fe-based superconductors
}

\author{
A.V. Chubukov ${ }^{1}$, D.V. Efremov ${ }^{2}$, and I. Eremin ${ }^{3,4}$ \\ 1 Department of Physics, University of Wisconsin-Madison, Madison, WI 53706, USA \\ 2 Institut für Theoretische Physik, Technische Universität Dresden, 01062 Dresden, Germany \\ 3 Max-Planck Institut für Physik komplexer Systeme, D-01187 Dresden, Germany \\ 4 Institute für Mathematische und Theoretische Physik, \\ TU-Braunschweig, D-38106 Braunschweig, Germany
}

(Dated: October 30, 2018)

\begin{abstract}
We analyze antiferromagnetism and superconductivity in novel $F e$-based superconductors within the itinerant model of small electron and hole pockets near $(0,0)$ and $(\pi, \pi)$. We argue that the effective interactions in both channels logarithmically flow towards the same values at low energies, i.e., antiferromagnetism and superconductivity must be treated on equal footings. The magnetic instability comes first for equal sizes of the two pockets, but looses to superconductivity upon doping. The superconducting gap has no nodes, but changes sign between the two Fermi surfaces (extended $s$-wave symmetry). We argue that the $T$ dependencies of the spin susceptibility and NMR relaxation rate for such state are exponential only at very low $T$, and can be well fitted by power-laws over a wide $T$ range below $T_{c}$.
\end{abstract}

PACS numbers: 74.20.Mn, 74.20.Rp, 74.25.Jb, 74.25.Ha

\section{INTRODUCTION}

Recent discovery of superconductivity in the ironbased layered pnictides with $T_{c}$ ranging between 26 and $52 \mathrm{~K}$ generated enormous interest in the physics of these materials [1, 2, 3, 4, 5]. The superconductivity has been discovered in oxygen containing $\mathrm{RFeAsO}(\mathrm{R}=\mathrm{La}$, $\mathrm{Nd}, \mathrm{Sm})$ as well as in oxygen free $\mathrm{AFe}_{2} \mathrm{As}_{2}(\mathrm{~A}=\mathrm{Ba}, \mathrm{Sr}$, $\mathrm{Ca}$ ). Like the cuprates, the pnictides are highly twodimensional, their parent material shows antiferromagnetic long-range order below $150 \mathrm{~K}$ [1, 6, 7, 8, 9], and superconductivity occurs upon doping of either electrons [1, 2, 3, 4] or holes [5] into the FeAs layers.

The close proximity of antiferromagnetism and superconductivity fueled early speculations that the physics of the pnictides is similar to the cuprates, and involves insulating behavior 10, 11, 12]. However, there is a growing consensus among researchers that Mott physics does not play a significant role for the iron pnictides, which remain itinerant for all doping levels, including parent compounds, in which magnetic order is of spindensity-wave (SDW) type rather than Heisenberg antiferromagnetism of localized spins 13, 14]. This is evidenced by, e.g., a relatively small value of the observed magnetic moment per Fe atom, which is around $12-16 \%$ of $2 \mu_{B}$ [7, 9]. In another distinction to the cuprates, electronic structure proposed by band structure calculations 15, 16, 17, 18, 19 and supported by ARPES 20, 21] consists of two small hole pockets centered around $\Gamma$ point $(\mathbf{p}=(0,0))$ and two small electron pockets centered around $M$ point $(\mathbf{p}=\mathbf{Q}=(\pi, \pi))$ in the folded Brillouin zone (BZ) (two $F e$ artoms in the unit cell, we set interatomic spacing $a=1$ )

In this paper, we address three issues for the pnictides: (i) what interactions cause SDW order and superconductivity, (ii) what is the gap symmetry, and (iii)

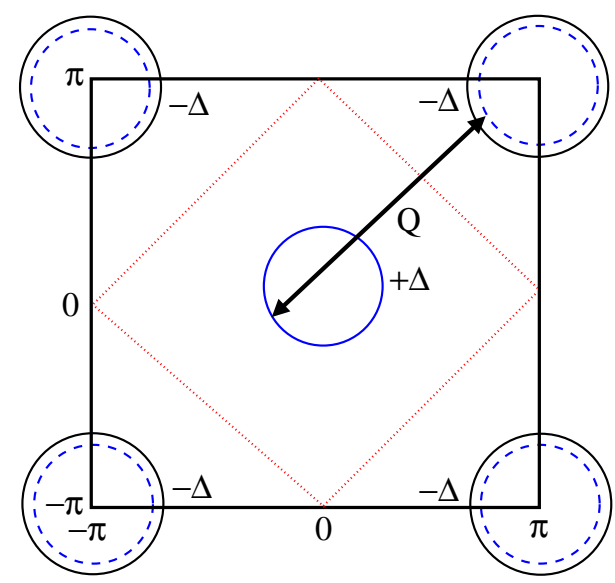

FIG. 1: (color online) A simplified FS geometry of doped Febased superconductors, used in the present work. At zero doping, the Fermi surface consists of an electron pocket around $(\pi, \pi)$ (black solid curve), and a hole pocket of roughly equal size around $(0,0)$ (blue solid curve). We neglect in this work the fact that there are two hole and two electron pockets. In this system, there is a near-perfect nesting between hole and electron pockets (moving a hole FS by $(\pi, \pi)$ one obtains a near-perfect match with an electron FS). Upon electron doping, the size of the electron pocket increases (dashed blue $\rightarrow$ black), and this breaks the nesting. $+\Delta$ and $-\Delta$ are the values of the superconducting gaps on the two FS for $s^{+}$superconducting state.

what are the implications of the gap symmetry for the experiments in the superconducting (SC) state. We argue that both magnetic and pairing instabilities are determined by the same interband pair hopping which transforms two fermions near the hole Fermi surface into two fermions near the electron Fermi surface (and vice versa). This interaction may be weak at high energies (of order bandwidth), but it flows under renorm-group (RG) and 
ultimately determines the couplings in both SDW and Cooper channels at low-energies. When electron and hole pockets are nearly identical, SDW instability occurs at a higher $T$. When the near-identity is broken by either hole or electron dopings, the Cooper instability comes first. This pairing interaction sets the gaps in hole and electron pockets to be of equal magnitude $\Delta$, but of opposite signs (an extended $s$-wave symmetry, $s^{+}$). The ratio $2 \Delta / T_{c}=3.53$ is, however, the same as in BCS theory, as there is no angular variations of the gap along the FS.

A fingerprint of $s^{+}$gap symmetry and near-equal electron and hole pockets is the existence of a magnetic collective mode inside the gap for momenta near $\mathbf{Q}$ (a spin resonance), whose dispersion $\left(\Omega_{0}^{2}+\left(v_{F}^{2} / 2\right)(\mathbf{q}-\mathbf{Q})^{2}\right)^{1 / 2}$, where $v_{F}$ is the Fermi velocity, closely resembles Anderson-Bogolubov mode in uncharged superconductors. Another fingerprint is a strong reduction of the nuclear magnetic resonance (NMR) relaxation rate $1 / T_{1}$ in the clean limit, due to vanishing of the coherence factor for $\chi^{\prime \prime}(\mathbf{q}, \omega) / \omega$ for $\mathbf{q}=\mathbf{Q}$. We argue that in this situation, $1 / T_{1}$ is predominantly due to impurities, which are partly pair-breaking even when non-magnetic. We show that, in the presence of impurity scattering, $1 / T_{1}$ is exponential in $T$ only for very low temperatures, and over a wide range of $T<T_{c}$ is well described by $1 / T_{1} \propto T^{3}$, as if the gap had nodes. Over the same range of $T$, the uniform susceptibility is near-linear in $T$.

Our results partly agree and partly disagree with some earlier works on Fe-pnictides. Mazin et al. [18] and Gorkov and Barzykin 22] conjectured that the pairing symmetry should be $s^{+}$. Our results agree with theirs and also with Eremin and Korshunov [23], who analyzed numerically the magnetic response at $\mathbf{Q}$ within RPA for an $s^{+}$superconductor and found the resonance peak be-

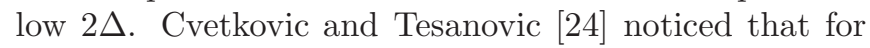
identical electron and hole pockets, Cooper and particlehole channels become undistinguishable and should be treated equally - the notion we share. Wang et al [25, 26] performed numerical RG study of the pairing symmetry and found an $s^{+}$gap symmetry for two-band model and a conventional $s$-wave symmetry for five-band model. We can only compare the results for the two-band model, for which we also found an attraction in in $s^{+}$channel. There is, however, an important difference between our results and those of Wang et al. In our case, the bare interaction in $s^{+}$channel is repulsive, and attraction emerges only below some energy scale, due to RG flow of the coupling. In their analysis, the bare interaction is zero, and attraction emerges already after an infinitesimal RG transformation. Lorenziana et al. 27] used unrestricted Hartree-Fock approximation and studied possible phases that may compete with superconductivity in FeAs layers. We found that SDW is the main competitor, but CDW with complex order parameter is close second.

On experimental side, ARPES 28, 29, 30] and Andreev spectroscopy 31] measurements have been interpreted as evidence for a nodeless gap, while NMR data were argued to follow $1 / T_{1} \propto T^{3}$ and were interpreted as evidence for a $d$-wave gap 32, 33] or multiple gaps 34. Our results show that the $T$ dependence of $1 / T_{1}$ in a dirty $s^{+}$superconductor mimics $T^{3}$ over a wide range of $T$ and become exponential only at very low temperatures.

\section{THE MODEL}

We model iron pnictides by an itinerant electron system with two electronic orbitals, and we assume that the hybridization between the orbitals leads to small hole and electron pockets located near $(0,0)$ and $(\pi, \pi)$, respectively in the folded BZ (two $\mathrm{Fe}$ atoms per unit cell) (Fig 1). The extension to a more realistic case of four (or even five) orbitals and two hole and two electron pockets is straightforward, and does not lead to new physics except for a magnetically ordered state, where four-pocket structure is essential for proper identification of relative magnetic ordering of spins of the two $\mathrm{Fe}$ atoms from the unit cell in folded BZ [26, 35, 36].

We assume that electron-electron interaction is shortrange (Hubbard-like) and involves two couplings - between fermionic densities from the same orbital and from different orbitals [37]. The Hamiltonian has the form $\mathcal{H}=\mathcal{H}_{2}+\mathcal{H}_{4}$, where

$$
\begin{aligned}
& \mathcal{H}_{2}=\sum_{\mathbf{p}, \sigma} \epsilon_{1, \mathbf{p}} \psi_{1, \mathbf{p}, \sigma}^{\dagger} \psi_{1, \mathbf{p}, \sigma}+\epsilon_{2, \mathbf{p}} \psi_{2, \mathbf{p}, \sigma}^{\dagger} \psi_{2, \mathbf{p}, \sigma}+\Gamma_{\mathbf{p}}\left(\psi_{1, \mathbf{p}, \sigma}^{\dagger} \psi_{2, \mathbf{p}, \sigma}+\psi_{2, \mathbf{p}, \sigma}^{\dagger} \psi_{1, \mathbf{p}, \sigma}\right) \\
& \mathcal{H}_{4}=\frac{U_{11}}{2} \sum_{\mathbf{p}_{i}, \sigma \neq \sigma^{\prime}}\left[\psi_{1, \mathbf{p}_{1}, \sigma}^{\dagger} \psi_{1, \mathbf{p}_{2}, \sigma} \psi_{1, \mathbf{p}_{3}, \sigma^{\prime}}^{\dagger} \psi_{1, \mathbf{p}_{4}, \sigma^{\prime}}+\psi_{2, \mathbf{p}_{1}, \sigma}^{\dagger} \psi_{2, \mathbf{p}_{2}, \sigma} \psi_{2, \mathbf{p}_{3}, \sigma^{\prime}}^{\dagger} \psi_{2, \mathbf{p}_{4}, \sigma^{\prime}}\right]+U_{12} \sum_{\mathbf{p}_{i}, \sigma, \sigma^{\prime}} \psi_{1, \mathbf{p}_{1}, \sigma}^{\dagger} \psi_{2, \mathbf{p}_{2}, \sigma} \psi_{2, \mathbf{p}_{3}, \sigma^{\prime}}^{\dagger} \psi_{2, \mathbf{p}_{4}, \sigma^{\prime}}
\end{aligned}
$$

where where $\mathbf{p}_{\mathbf{1}}+\mathbf{p}_{\mathbf{2}}=\mathbf{p}_{\mathbf{3}}+\mathbf{p}_{\mathbf{4}}$, and $U_{11}$ is intra-orbital,

and $U_{12}$ inter-orbital interactions which we approximate 

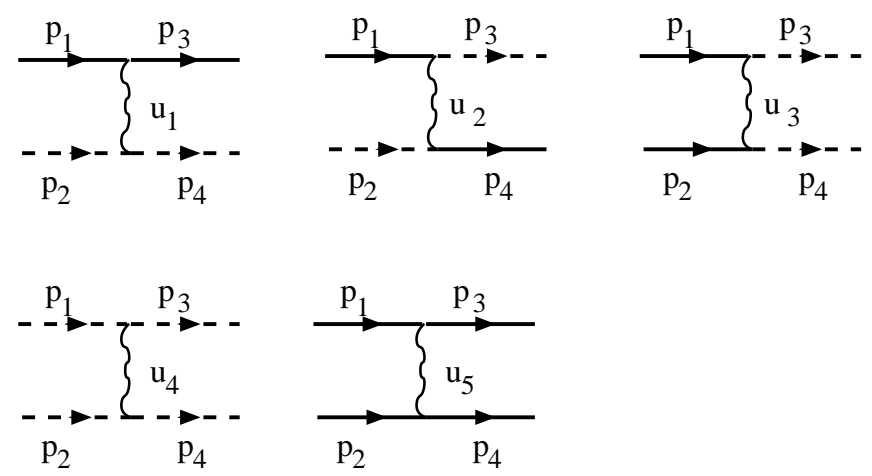

FIG. 2: Five relevant bare vertices. Solid and dashed lines represent fermions from $c$ - band (near $\mathbf{k}=0$ ) and $f$-band $($ near $\mathbf{k}=(\pi, \pi))$.

by momentum-independent (on-site) values.

The quadratic form can be easily diagonalized by

$$
\begin{aligned}
& \psi_{1, \mathbf{p}, \sigma}=\cos \theta_{\mathbf{p}} c_{\mathbf{p}, \sigma}+\sin \theta_{\mathbf{p}} f_{\mathbf{p}, \sigma} \\
& \psi_{2, \mathbf{p}, \sigma}=\cos \theta_{\mathbf{p}} f_{\mathbf{p}, \sigma}-\sin \theta_{\mathbf{p}} c_{\mathbf{p}, \sigma}
\end{aligned}
$$

with $\tan 2 \theta_{\mathbf{p}}=2 \Gamma_{\mathbf{p}} /\left(\epsilon_{2, \mathbf{p}}-\epsilon_{1, \mathbf{p}}\right)$. This yields

$$
\mathcal{H}_{2}=\sum_{\mathbf{p}, \sigma} \epsilon_{\mathbf{p}}^{c} c_{\mathbf{p}, \sigma}^{\dagger} c_{\mathbf{p}, \sigma}+\epsilon_{\mathbf{p}}^{f} f_{\mathbf{p}, \sigma}^{\dagger} f_{\mathbf{p}, \sigma}
$$

where

$$
\epsilon_{\mathbf{p}}^{c, f}=\frac{\epsilon_{1, \mathbf{p}}+\epsilon_{2, \mathbf{p}}}{2} \mp \frac{1}{2} \sqrt{\left(\epsilon_{1, \mathbf{p}}-\epsilon_{2, \mathbf{p}}\right)^{2}+4 \Gamma_{\mathbf{p}}^{2}}
$$

Under some conditions on the original dispersions $\epsilon_{1, \mathbf{p}}$ and $\epsilon_{2, \mathbf{p}}$, and on the hybridization term $\Gamma_{\mathbf{p}}$, the two bands of fermionic excitations form small hole and electron pockets near $(0,0)$ and $\mathbf{Q}=(\pi, \pi)$, with roughly equal size, as in the iron pnictides. This happens if, e.g., $\epsilon_{1, \mathbf{p}}$ and $\epsilon_{2, \mathbf{p}}$ change sign under $\mathbf{p} \rightarrow \mathbf{p}+\mathbf{Q}, \epsilon_{1, \mathbf{0}}+\epsilon_{2, \mathbf{0}}>0$, $\Gamma_{\mathbf{p}+\mathbf{Q}}= \pm \Gamma_{\mathbf{p}}$, and $\sqrt{\left(\epsilon_{1, \mathbf{p}}-\epsilon_{2, \mathbf{p}}\right)^{2}+4 \Gamma_{\mathbf{p}}^{2}} \leq\left|\epsilon_{1, \mathbf{p}}+\epsilon_{2, \mathbf{p}}\right|$. Under these conditions, $\epsilon_{\mathbf{p}}^{c}=-\epsilon_{\mathbf{p}+\mathbf{Q}}^{f}$, and $\epsilon_{\mathbf{p}}^{c}$ describes a hole band with the maximum of energy at $(0,0)$, while $\epsilon_{\mathbf{p}}^{f}$ describes an equivalent electron band with the minimum of energy at $\mathbf{Q}$. Upon doping, chemical potential shifts, one Fermi surface gets larger while the other gets smaller, see Fig.1.

In itinerant systems, the interactions are expected to be small compared to the fermionic bandwidth, and the physics is dominated by fermions near the Fermi surface (FS). The projection of the Hubbard interaction term $\mathcal{H}_{4}$ onto $c$ and $f$ fermions leads to five different interactions:

$$
\begin{aligned}
& H_{4}=U_{1}^{(0)} \sum c_{\mathbf{p}_{3} \sigma}^{\dagger} f_{\mathbf{p}_{4} \sigma^{\prime}}^{\dagger} f_{\mathbf{p}_{2} \sigma^{\prime}} c_{\mathbf{p}_{1} \sigma}+U_{2}^{(0)} \sum f_{\mathbf{p}_{3} \sigma}^{\dagger} c_{\mathbf{p}_{4} \sigma^{\prime}}^{\dagger} f_{\mathbf{p}_{2} \sigma^{\prime}} c_{\mathbf{p}_{1} \sigma} \\
& +\frac{U_{3}^{(0)}}{2} \sum\left[f_{\mathbf{p}_{3} \sigma}^{\dagger} f_{\mathbf{p}_{4} \sigma^{\prime}}^{\dagger} c_{\mathbf{p}_{2} \sigma^{\prime}} c_{\mathbf{p}_{1} \sigma}+h . c\right]+\frac{U_{4}^{(0)}}{2} \sum f_{\mathbf{p}_{3} \sigma}^{\dagger} f_{\mathbf{p}_{4} \sigma^{\prime}}^{\dagger} f_{\mathbf{p}_{2} \sigma^{\prime}} f_{\mathbf{p}_{1} \sigma}+\frac{U_{5}^{(0)}}{2} \sum c_{\mathbf{p}_{3} \sigma}^{\dagger} c_{\mathbf{p}_{4} \sigma^{\prime}}^{\dagger} c_{\mathbf{p}_{2} \sigma^{\prime}} c_{\mathbf{p}_{1} \sigma}
\end{aligned}
$$

where the momenta of $c$ - fermions are near $(0,0)$, the momenta of $f$-fermions are near $(\pi, \pi)$, and the momentum conservation is assumed. We present these interactions graphically in Fig 2

We label the couplings with subindex " 0 " to emphasize that these are the bare couplings. The terms with $U_{4}^{(0)}$ and $U_{5}^{(0)}$ are intraband interactions, the terms with $U_{1}^{(0)}$ and $U_{2}^{(0)}$ are interband interactions with momentum transfer 0 and $\mathbf{Q}$, respectively, and the term with $U_{3}^{(0)}$ is interband pair hopping.

Note that in our Fermi-liquid description, all vertices in Eq. (5) are $\delta$-functions in spin indices, i.e., all interactions are in the charge channel [38], and there are no direct spin-spin interaction terms with spin matrices in the vertices. However, if the original Hubbard interaction is on-site, one can use another, equivalent, description in which Pauli principle is build into the Hamiltonian, and the intra-orbital terms with equal spin projections are eliminated from the Hamiltonian. In this description, $U_{1}^{(0)}, U_{4}^{(0)}$, and $U_{5}^{(0)}$ terms appear as effective Hubbard interactions, while $U_{2}^{(0)}$ and $U_{3}^{(0)}$ appear as a magnetic, Hund term 25].

In explicit form, $U_{i}^{(0)}$ are

$$
\begin{aligned}
& U_{1}^{(0)}=\frac{1}{2}\left[\left(U_{11}+U_{12}\right)-\cos 2 \theta_{0} \cos 2 \theta_{\mathbf{Q}}\left(U_{11}-U_{12}\right)\right] \\
& U_{2,3}^{(0)}=\frac{U_{11}}{2}\left(1-\cos 2 \theta_{0} \cos 2 \theta_{\mathbf{Q}}\right)-\frac{U_{12}}{2} \sin 2 \theta_{0} \sin 2 \theta_{\mathbf{Q}} \\
& U_{4}^{(0)}=\frac{U_{11}+U_{12}}{2}+\frac{U_{11}-U_{12}}{2} \cos ^{2} 2 \theta_{0}, \\
& U_{5}^{(0)}=\frac{U_{11}+U_{12}}{2}+\frac{U_{11}-U_{12}}{2} \cos ^{2} 2 \theta_{\mathbf{Q}},
\end{aligned}
$$

For the case that we considered above $\left(\epsilon_{1, \mathbf{p}}=\right.$ $\left.-\epsilon_{1, \mathbf{p}+\mathbf{Q}}, \epsilon_{2, \mathbf{p}}=-\epsilon_{2, \mathbf{p}+\mathbf{Q}}, \Gamma_{\mathbf{p}+\mathbf{Q}}= \pm \Gamma_{\mathbf{p}}\right)$, we have $\theta_{\mathbf{Q}}=$ $\pi / 2 \mp \theta_{0}$, we have

$$
\begin{aligned}
& U_{1}^{(0)}=U_{4}^{(0)}=U_{5}^{(0)}=\frac{U_{11}+U_{12}}{2}+\frac{U_{11}-U_{12}}{2} \cos ^{2} 2 \theta_{0}, \\
& U_{2}^{(0)}=U_{3}^{(0)}=\frac{U_{11}}{2}\left(1+\cos ^{2} 2 \theta_{0}\right) \mp \frac{U_{12}}{2} \sin ^{2} 2 \theta_{0}
\end{aligned}
$$


We assume that the intra and inter-orbital Hubbard-type interactions $U_{11}$ and $U_{12}$ are positive (repulsive). We see from (77) that density-density couplings $U_{1}^{(0)}, U_{4}^{(0)}$, and $U_{5}^{(0)}$ are positive and the largest. The couplings $U_{2}^{(0)}$ and $U_{3}^{(0)}$ are smaller for the case when the hybridization term is even under $\mathbf{p} \rightarrow \mathbf{p}+\mathbf{Q}$, i.e., $\Gamma_{\mathbf{p}}=\Gamma_{\mathbf{p}+\mathbf{Q}}$, and are the same as $U_{1}^{(0)}, U_{4}^{(0)}$, and $U_{5}^{(0)}$ when $\Gamma_{\mathbf{p}}=$ $\Gamma_{\mathbf{p}+\mathbf{Q}}$. The first case corresponds to on-site hybridization and is more realistic that the second one, which requires hybridization to involve predominantly nearest neighbors. Below we will consider only the first case $\Gamma_{\mathbf{p}}=\Gamma_{\mathbf{p}+\mathbf{Q}}$. Note that in this situation, the sign of $U_{2}^{(0)}=U_{3}^{(0)}$ depends on $\theta_{0}$ and on the relative strength of the intra-orbital and inter-orbital Hubbard terms. If $U_{11}>U_{12} \sin ^{2} 2 \theta_{0} /\left(1+\cos ^{2} 2 \theta_{0}\right)$, these couplings are positive, if $U_{11}<U_{12} \sin ^{2} 2 \theta_{0} /\left(1+\cos ^{2} 2 \theta_{0}\right)$, they are negative. A more likely situation is when the intra-orbital Hubbard term $U_{11}$ is larger than inter-orbital $U_{12}$, in which case $U_{2}^{(0)}$ and $U_{3}^{(0)}$ are positive.

For convenience, below we will be using dimensionless interactions

$$
u_{i}=U_{i} N_{0}
$$

where $N_{0}$ is the fermionic density of states (DOS) which we approximate by a constant. For itinerant systems, $\left|u_{i}^{0}\right|<1$ and can be treated within Fermi liquid theory. We will also count the momenta of $f$-fermions as deviations from $\mathbf{Q}\left(f_{\mathbf{p}} \rightarrow f_{\mathbf{p}+\mathbf{Q}}\right)$ in which case all running momenta in the vertices are small.

\section{Density wave and pairing instabilities}

We searched for possible density-wave and Cooperpairing instabilities for our model, and found that the ones which may potentially occur are spin density wave (SDW) and charge density wave (CDW) instabilities with momentum $\mathbf{Q}$ and with either real or imaginary order parameter, and superconducting (SC) instability either in pure $s$ channel (the gaps $\Delta_{c}$ and $\Delta_{f}$ have the same sign), or in $s^{+}$channel (the gaps $\Delta_{c}$ and $\Delta_{f}$ have opposite sign). Density-wave instabilities with $\mathbf{q}=0$ and pairing instabilities with $\mathbf{q}=\mathbf{Q}$ do not occur within our model because the corresponding kernels vanish for a constant DOS. The instabilities with momentum-dependent order parameter, like a nematic instability [35] also do not occur simply because we set all interactions to be momentum-independent and weak, and will neglect regular (non-logarithmic) corrections which give rise to the momentum dependence of the scattering amplitude in a Fermi liquid [38].

The temperatures of potential density-wave and pairing instabilities are obtained by conventional means, by
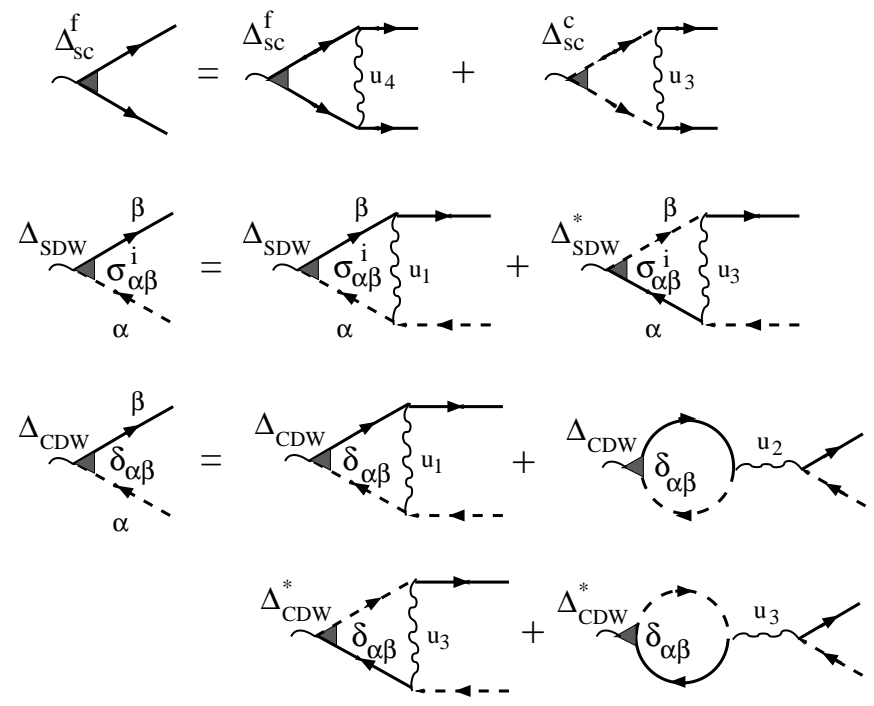

FIG. 3: Diagrammatic representation of the equations for SDW, CDW, and SC instability temperatures. The equations for density-wave $T_{s d w}^{(r, i)}$ and $T_{c d w}^{(r, i)}$ are obtained by adding and and subtracting equations for $\Delta_{s d w}$ and $\Delta_{s d w}^{*}$, and for $\Delta_{c d w}$ and $\Delta_{c d w}^{*}$, respectively. The equations for $T_{s c}^{(s)}$ and $T_{s c}^{\left(s^{+}\right)}$obtained by adding and and subtracting equations for $\Delta^{c}$ and $\Delta^{f}$.

introducing infinitesimal couplings

$$
\begin{aligned}
& \Delta_{s d w} \sum_{k} c_{\mathbf{k}, \alpha}^{\dagger} \sigma_{\alpha \beta}^{z} f_{\mathbf{k}+\mathbf{Q}, \beta}, \\
& \Delta_{c d w} \sum_{k} c_{\mathbf{k}, \alpha}^{\dagger} \delta_{\alpha \beta} f_{\mathbf{k}+\mathbf{Q}, \beta}, \\
& \Delta_{s c}^{c} \sum_{k} c_{\mathbf{k}, \alpha} \sigma_{\alpha \beta}^{y} c_{-\mathbf{k}, \beta}+\Delta_{s c}^{f} \sum_{k} f_{\mathbf{k}+\mathbf{Q}, \alpha} \sigma_{\alpha \beta}^{y} f_{-\mathbf{k}-\mathbf{Q}, \beta}
\end{aligned}
$$

with complex $\Delta_{s d w}, \Delta_{c d w}$, and real $\Delta_{s c}^{c, f}$, and analyzing when the response functions diverge. We label the corresponding instability temperatures as $T_{s d w}^{(r, i)} T_{c d w}^{(r, i)}$ and $T_{s c}^{\left(s, s^{+}\right)}$, where $r, i$ mean real or imaginary density-wave order parameter, and $s, s^{+}$mean $s$-wave or extended $s$-wave, respectively. The linearized equations for the order parameters are presented graphically in Fig 3 They have non-zero solutions when

$$
\begin{aligned}
& 1=-T_{s d w}^{(r, i)} \sum_{\omega_{m}} \Gamma_{s d w}^{(r, i)} \int d \epsilon_{\mathbf{k}} G_{\mathbf{k} \omega_{m}}^{c} G_{\mathbf{k}+\mathbf{Q}, \omega_{m}}^{f} \\
& 1=-T_{c d w}^{(r, i)} \sum_{\omega_{m}} \Gamma_{c d w}^{(r, i)} \int d \epsilon_{\mathbf{k}} G_{\mathbf{k} \omega_{m}}^{c} G_{\mathbf{k}+\mathbf{Q}, \omega_{m}}^{f} \\
& 1=-T_{s c}^{\left(s, s^{+}\right)} \sum_{\omega_{m}} \Gamma_{s c}^{\left(s, s^{+}\right)} \int d \epsilon_{\mathbf{k}} G_{\mathbf{k} \omega_{m}}^{c} G_{-\mathbf{k},-\omega_{m}}^{c}
\end{aligned}
$$

Here

$$
\begin{aligned}
\Gamma_{s d w}^{(r, i)}=u_{1} \pm u_{3}, & \Gamma_{c d w}^{(r, i)}=u_{1} \mp u_{3}-2 u_{2}, \\
\Gamma_{s c}^{(s)}=u_{4}+u_{3}, & \Gamma_{s c}^{\left(s^{+}\right)}=u_{4}-u_{3}
\end{aligned}
$$


are the full interactions in the SDW, CDW, and SC channels. Eq. (10) is only valid for the largest instability temperature. Below such $T$, the ordering in one channel affects susceptibilities in the other channels.

For the bare parameters as in (7)

$$
\begin{aligned}
& \Gamma_{s d w}^{(r)}=u_{1}^{0}+u_{3}^{0} \approx u_{11}\left(1+\cos ^{2} 2 \theta_{0}\right), \\
& \Gamma_{s d w}^{(i)}=u_{1}^{0}-u_{3}^{0} \approx u_{12} \sin ^{2} 2 \theta_{0} \\
& \Gamma_{c d w}^{(r)}=u_{1}^{0}-u_{3}^{0}-2 u_{2}^{0} \approx 2 u_{12} \sin ^{2} 2 \theta_{0}-u_{11}\left(1+\cos ^{2} 2 \theta_{0}\right), \\
& \Gamma_{c d w}^{(i)}=u_{1}^{0}+u_{3}^{0}-2 u_{2}^{0} \approx u_{12} \sin ^{2} 2 \theta_{0} \\
& \Gamma_{s c}^{(s)}=u_{4}^{0}+u_{3}^{0} \approx u_{11}\left(1+\cos ^{2} 2 \theta_{0}\right), \\
& \Gamma_{s c}^{\left(s^{+}\right)}=u_{4}^{0}-u_{3}^{0} \approx u_{12} \sin ^{2} 2 \theta_{0}
\end{aligned}
$$

where $u_{11}=U_{11} N_{0}, u_{12}=U_{12} N_{0}$ are dimensionless intra-orbital and inter-orbital Hubbard couplings. The Stoner-like SDW and CDW instabilities require $\Gamma_{s d w}, \Gamma_{c d w}>0$. At the bare level, $\Gamma_{s d w}^{(r)}$ is the largest positive interaction when $u_{11}\left(1+\cos ^{2} 2 \theta_{0}\right)>u_{12} \sin ^{2} 2 \theta_{0}$ and $\Gamma_{c d w}^{(r)}$ is the largest positive interaction when $u_{11}(1+$ $\left.\cos ^{2} 2 \theta_{0}\right)<u_{12} \sin ^{2} 2 \theta_{0}$ i.e., the system undergoes a conventional SDW or CDW instability. The SC instabilities requires an attraction (a negative $\Gamma_{s c}^{\left(s, s^{+}\right)}$) and do not occur at this level because both $\Gamma_{s c}^{(s)}$ and $\Gamma_{s c}^{\left(s^{+}\right)}$are positive.

\section{RG FLOW}

Beyond mean-field, the potential SDW and SC instabilities are determined by $u_{i}$ at energies below the Fermi energy $E_{F}$, and generally differ from bare $u_{i}^{0}$ defined at energies comparable to the bandwidth, $W$. For small size of the FS, $W>>E_{F}$, and the intermediate range is quite large. At $u_{i}^{0}<1$ the renormalization can be considered in one-loop approximation. The one-loop diagrams, shown in Fig 4, contain particle-particle and particle-hole bubbles. The external momenta in these diagrams are of order running $E \geq E_{F}$, while internal momenta are generally of order $W$, i.e., much larger. In this situation, the dependence on the directions of the external momenta is lost, i.e., a SC vertex with zero total momentum and an SDW vertex with transferred momentum $\mathbf{Q}$ are renormalized in the same way. The crucial element of our analysis is the observation that, for $\epsilon_{\mathbf{p}}^{c}=-\epsilon_{\mathbf{p}+\mathbf{q}}^{f}$, particle-hole channel is undistinguishable from particleparticle channel, such that the renormalization in both channels are logarithmical and interfere with each other. The presence of the logarithms in both channels implies that the one-loop perturbation theory must be extended to one loop RG analysis for the running $u_{i}$ (in the diagrammatic language, one needs to sum up series of logarithmically divergent parquet diagrams). The derivation of the RG equations is straightforward (see Fig. (4). Collecting combinatoric pre-factors for the diagrams, we (a)
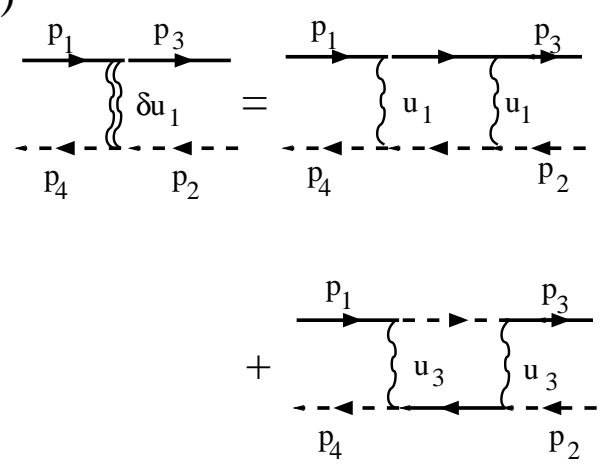

(b)
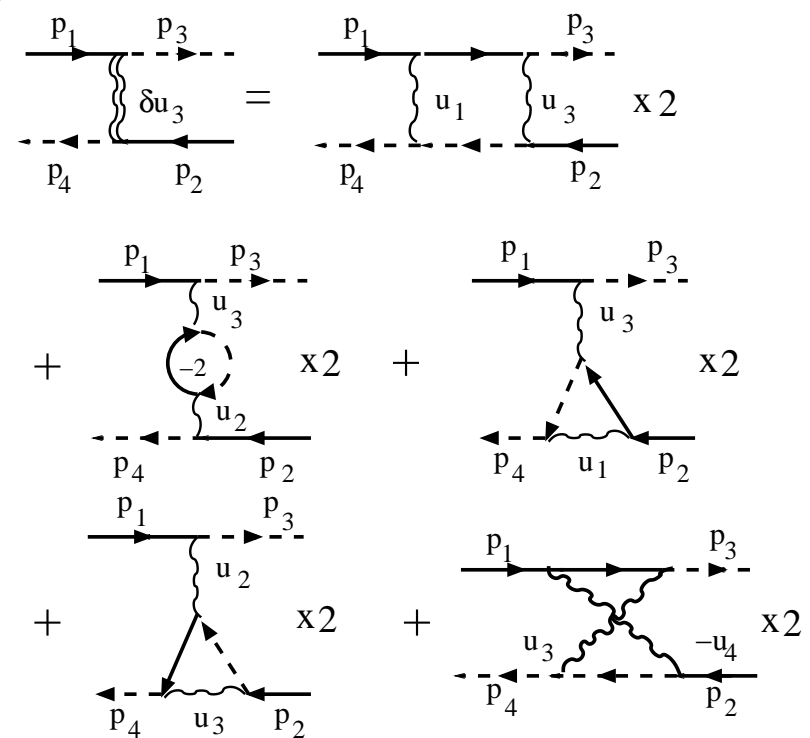

FIG. 4: Diagrams for one-loop vertex renormalizations. The renormalizations of $u_{1}$ and $u_{3}$ are shown, others are obtained in a similar way.

obtain

$$
\begin{aligned}
& \dot{u}_{1}=u_{1}^{2}+u_{3}^{2} \\
& \dot{u}_{2}=2 u_{2}\left(u_{1}-u_{2}\right) \\
& \dot{u}_{3}=2 u_{3}\left(2 u_{1}-u_{2}-u_{4}\right) \\
& \dot{u}_{4}=-u_{3}^{2}-u_{4}^{2}
\end{aligned}
$$

where the derivatives are with respect to $\log W / E$, and $E$ is the running energy scale. Similar, though not identical equations have been obtained in the weak-coupling studies of the cuprates with the " $t$-only" dispersion [39].

We see from Eq.(13) that the pair hopping term $u_{3}$ is not generated by other interactions, i.e., $u_{3}=0$ if $u_{3}^{0}=0$. In the absence of $u_{3}, \Gamma_{s d w}$ and $\Gamma_{s c}^{s, s^{+}}-\Gamma_{s d w}=u_{1}$ increases and drives $T_{s d w}$ up, while $\Gamma_{s c}^{s, s^{+}}=u_{4}$ logarithmically decreases, as it is expected for a repulsive interaction [40]. However, once $u_{3}^{0}$ is finite, the system moves into the basin of attraction of another fixed point, at 


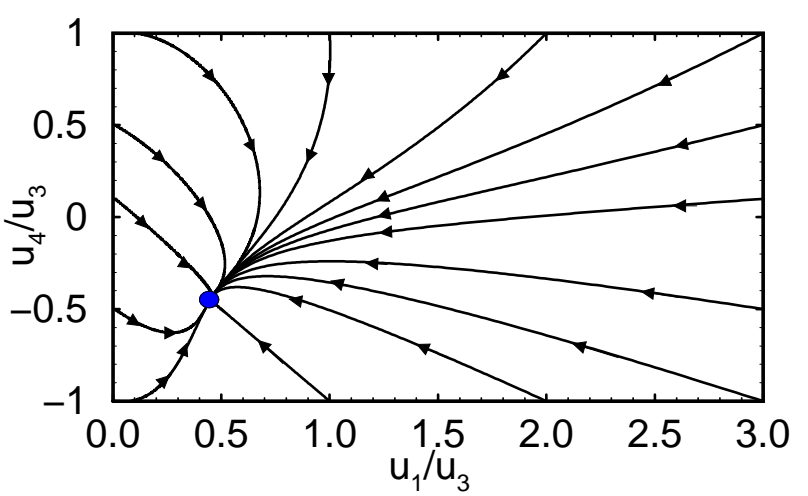

FIG. 5: (color online) The RG flow of Eqn. (13) in variables $u_{4} / u_{3}$ and $u_{1} / u_{3}$. The fixed point is $u_{4} / u_{3}=$ $-1 / \sqrt{5}, \quad u_{1} / u_{3}=1 / \sqrt{5}$. The fourth variable, $u_{2}$, becomes small near the fixed point compared to the other $u_{i}$, We used boundary conditions $u_{1}^{0}=u_{4}^{0}, u_{3}^{0}=0.1 u_{1}^{0}$, and for simplicity set $u_{0}^{2}=0$.

which

$$
u_{3} \propto \frac{u}{1-|u| \ln \frac{W}{E}}, \quad u_{1}=-u_{4}=\frac{\left|u_{3}\right|}{\sqrt{5}}, u_{2} \propto\left|u_{3}\right|^{1 / 3}
$$

where $u$ depends on the bare values of the couplings. In Figs 5 and 6 we show the RG flow obtained by the numerical solution of Eq. (13).

The two key features of the new fixed point are (i) $\left|u_{3}\right|$ rapidly increases and eventually becomes larger than $u_{1}$ by a factor $\sqrt{5}$, and (ii) $u_{4}$ decreases, passes through zero, changes sign, and then increases by magnitude and approaches $-\left|u_{3}\right| / \sqrt{5}$ (see Fig. 5)

For positive $u_{3}^{0} \approx u_{2}^{0}$, these results imply that $\Gamma_{s d w}^{r}=$ $u_{1}+u_{3}$ remains positive and the largest out of densitywave vertices i.e., the highest density-wave instability is a conventional SDW instability (see Fig. 66). Note, however, that $\Gamma_{c d w}^{(i)}$ is close second as it only differs by $u_{2}$ which under renormalization becomes relatively small compared to $u_{1}$ and $u_{3}\left(u_{2} \propto\left(u_{3}\right)^{1 / 3}\right)$. The interaction in the $s^{+}$SC channel, $\Gamma_{s c}^{(s+)}=u_{4}-u_{3}$, becomes negative (attractive) below some scale (Fig. 6a), while $\Gamma_{s c}^{(s)}$ remains repulsive. We emphasize that the density-density vertex $u_{4}$ changes sign under renormalization, becomes attractive and also supports SC. Moreover, the interactions in the SDW and the $s+\mathrm{SC}$ channel $\Gamma_{s d w}^{(r)}=u_{1}+u_{3}$ and $\Gamma_{s c}^{(s+)}=u_{3}-u_{4}$, become comparable to each other and eventually flow to the same value $u_{3}(1+1 / \sqrt{5})$. The implication is that the SDW order and $s^{+}$superconductivity are competing orders, determined by effective interactions of comparable strength.

For negative $u_{3}^{0}, u_{2}^{0}, \Gamma_{c d w}^{(r)}=u_{1}+\left|u_{3}\right|+2\left|u_{2}\right|$ is the strongest, positive, density-wave vertex, and $\Gamma_{s d w}^{(i)}=$ $u_{1}+\left|u_{3}\right|$ is a close second (see Fig. 6 b). $\Gamma_{s c}^{\left(s^{+}\right)}=u_{4}+\left|u_{3}\right|$ is now positive (repulsive), but $\Gamma_{s c}^{(s)}=u_{4}-\left|u_{3}\right|$, changes
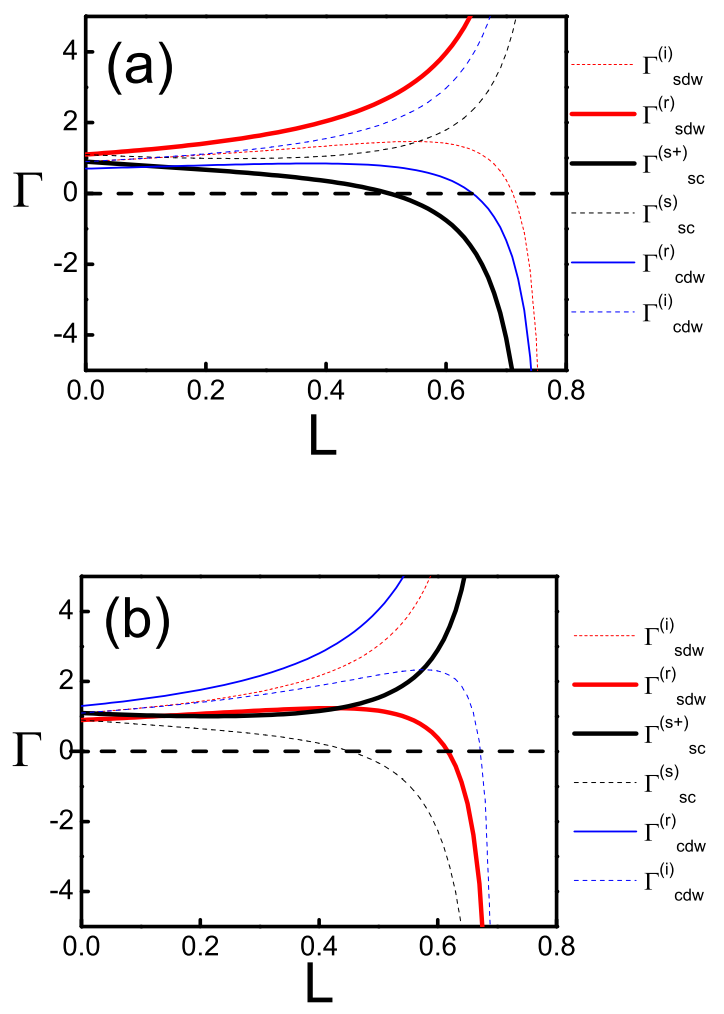

FIG. 6: (color online) The RG flow of the effective couplings in various density-wave and superconducting channels vs $L=$ $u^{0} \log W / E$. The boundary conditions are $u_{1}^{0}=u_{4}^{0}=u_{0}$, and $u_{2}^{0}=u_{3}^{0}= \pm 0.1 u_{0}$. The running couplings are in units of $u_{0}$. Panel a) - the RG flow for $u_{3}^{0}>0$. The extended $s$-wave $\left(s^{+}\right)$superconducting channel becomes attractive above some $L$. The strongest density-wave instability is in SDW channel, for real order parameter. Panel b) - the same for $u_{3}^{0}<0$. The conventional $s$-wave superconducting channel becomes attractive above some $L$. The strongest density-wave instability is in CDW channel, again for real order parameter.

sign under the renormalization and becomes negative (attractive), see Fig. 6b. This implies that CDW now competes with a conventional $s$-wave SC. Near the fixed point, the interaction in the $s$-channel $\Gamma_{s c}^{(s)} \approx-\left|u_{3}\right|(1+$ $1 / \sqrt{5})$ is now larger than $\Gamma_{c d w}^{(r)} \approx\left|u_{3}\right|(1-1 / \sqrt{5})$ which implies that in this case $s$-wave SC likely wins over CDW.

The generalization of this analysis to 4-band model (or even five) is straightforward and yields qualitatively similar behavior.

\section{Competing orders}

We next analyze in more detail Eqs. (10) for $u_{3}^{0}>0$. By construction, the upper limit of the integration over internal energies there is $O\left(E_{F}\right)$ as the contributions from 
higher energies are already absorbed into the renormalized vertices. When hole and electron Fermi surfaces are near-identical, i,e., $\epsilon_{\mathbf{k}}^{c}=-\epsilon_{\mathbf{k}+\mathbf{Q}}^{f}$ holds down to the lowest energies, both SDW and SC susceptibilities are logarithmic in $T$

$$
\begin{aligned}
& -T \sum_{\omega} \int d \epsilon_{\mathbf{k}} G_{\mathbf{k} \omega_{m}}^{c} G_{\mathbf{k}+\mathbf{Q}, \omega_{m}}^{f}=T \sum_{\omega} \int d \epsilon_{\mathbf{k}} G_{\mathbf{k} \omega_{m}}^{c} G_{-\mathbf{k},-\omega_{r}}^{c} \\
& =\int_{0}^{E_{F}} \tanh \left(\frac{\omega}{2 T}\right) \frac{d \omega}{\omega}=\log \frac{E_{F}}{T}
\end{aligned}
$$

and from (10) the largest instability temperature is either

$$
T_{s d w}^{(r)} \sim E_{F} e^{-\frac{1}{\Gamma_{s d w}^{(r)}}}, \quad \text { or } \quad T_{s c}^{\left(s^{+}\right)} \sim E_{F} e^{-\frac{1}{\left|\Gamma_{s c}^{(s+)}\right|}} .
$$

As $\Gamma_{s d w}^{(r)}$ is still larger than $\Gamma_{s c}^{\left(s^{+}\right)}$, the SDW instability comes first. This is what, we believe, happens at zero doping. Whether SC emerges as an extra order at a smaller $T$ requires a separate analysis as the pairing susceptibility changes in the presence of the SDW order. At a finite doping, all evidence is that the two FS become unequal, i.e., the condition $\epsilon_{\mathbf{k}}^{c}=-\epsilon_{\mathbf{k}+\mathbf{Q}}^{f}$ breaks down. In this situation, the $\log 1 / T$ behavior of the SDW polarization is cut, and $T_{s d w}^{(r)}$ decreases and eventually becomes smaller than $T_{c}^{\left(s^{+}\right)}$. At larger dopings, $T_{c}^{\left(s^{+}\right)}$remains roughly doping independent, while magnetic correlations decrease.

A remark about the SDW state. In the coordinate frame associated with folded BZ, $F$ e ions are located at $\mathbf{r}_{1}=\left(n_{x}, n_{y}\right)$, where $n_{x}, n_{y}$ are integers (we recall that we set interatomic spacing to one), but also at $\mathbf{r}_{2}=\left(n_{x}+\right.$ $\left.1 / 2, n_{y}+1 / 2\right)$. SDW instability with $\mathbf{Q}=(\pi, \pi)$ order antiferromagnetically spins within the sublattice where $\mathbf{r}=\mathbf{r}_{1}$, and within the sublattice where where $\mathbf{r}=\mathbf{r}_{2}$, but do not fix relative orientation between the spins in the two sublattices. To obtain full spin structure, we would need to analyze spin ordering within full four-band structure (two electron and and two hole orbitals), or go back into unfolded Brillouin zone. For localized spins, this type of order is described by $J_{1}-J_{2}$ model for $J_{2}>$ $0.5 J_{1}$. In the classical model, the angle between $\mathbf{r}_{1}$ and $\mathbf{r}_{2}$ sublattices is arbitrary, but quantum fluctuations select $(0, \pi)$ or $(\pi, 0)$ state [41, 42]. There is then an extra Ising degree of freedom, which was argued 35, 42 to remain broken even at $T>T_{s d w}$, when $S U(2)$ spin symmetry is restored.

\section{SUPERCONDUCTING STATE}

The SC $s^{+}$state that we found has two features similar to a conventional isotropic $s$-wave state. First, the superconducting gaps on the hole and electron FS are opposite in sign, but equal in magnitude. They, however, become unequal when $E_{F}$ on the two FS become is different, which happens once the doping increases (or when intraband density-density interactions $u_{4}$ and $u_{5}$ become unequal). Second, solving the non-linear gap equation, we immediately find that the gap $\Delta$ obeys the same BCS relation $2 \Delta=3.53 T_{c}$ as for an isotropic $s$-wave state simply because the pairing kernel contains either two $c$-fermions or two $d$-fermions, but no $c f$ pairs.

The $s^{+}$and $s$ SC states, however, differ qualitatively in the presence of non-magnetic impurities. For $s$-state, non-magnetic impurities do not affect $T_{c}$ and non-linear gap equation [43]. For $s^{+}$state, the impurity potential $U_{i}(\mathbf{q})$ has intra and interband components $U_{i}(0)$ and $U_{i}(\pi)$, respectively. The $U_{i}(\pi)$ components scatter fermions with $+\Delta$ and $-\Delta$ and acts as a "magnetic impurity" [44, 45]. Specifically, for the $s^{+}$state, normal and anomalous Greens functions in the presence of impurities are

$$
\begin{aligned}
G_{\mathbf{k}, \omega_{m}}^{c, f} & =\frac{Z_{\omega_{m}} \omega_{m} \pm \epsilon_{\mathbf{k}}}{Z_{\omega_{m}}^{2}\left(\omega_{m}^{2}+\bar{\Delta}_{\omega_{m}}^{2}\right)+\epsilon_{\mathbf{k}}^{2}} \\
F_{\mathbf{k}, \omega_{m}}^{c, f} & = \pm \frac{Z_{\omega_{m}} \bar{\Delta}_{\omega_{m}}}{Z_{\omega_{m}}^{2}\left(\omega_{m}^{2}+\bar{\Delta}_{\omega_{m}}^{2}\right)+\epsilon_{\mathbf{k}}^{2}},
\end{aligned}
$$

and the fermionic $Z=1+\Sigma\left(\omega_{m}\right) / \omega_{m}$ and the renormalized gap $\bar{\Delta}_{\omega_{m}}$ in the Born approximation are given by

$$
\begin{aligned}
& Z=1+\frac{U_{i}(0)+U_{i}(\pi)}{\sqrt{\Delta^{2}+\omega_{m}^{2}}} \\
& \frac{\bar{\Delta}_{\omega_{m}}}{\Delta}-1=-\frac{b_{T} \bar{\Delta}_{\omega_{m}}}{\sqrt{\bar{\Delta}_{\omega_{m}}^{2}+\omega_{m}^{2}}}
\end{aligned}
$$

where $\Delta=\Delta(T)$ is the frequency-independent order parameter, and $b_{T}=2 U_{i}(\pi) / \Delta(T)$. Below we use $b_{T=0}=b$ as a measure of the strength of impurity scattering. Note that $b$ is a complex function of the impurity strength as the order parameter is also affected by impurities (see below).

For $U_{i}(\pi)=0, \bar{\Delta}=\Delta$, i.e., superconductivity is not influenced by impurities. For $U_{i}(\pi) \neq 0, \bar{\Delta}_{\omega_{m}}$ becomes frequency dependent, as if the impurities were magnetic. At $T=0$, and $b \geq 1$, the system displays gapless superconductivity[46]: in real frequencies $\bar{\Delta}_{\omega_{m}} \propto-i \omega$ at small $\omega$, and the DOS at zero energy acquires a finite value $N(\omega=0)=\left(1-(1 / b)^{2}\right)^{1 / 2}$. Superconductivity at $T=0$ eventually disappears when $\Delta$ vanishes, i.e., when $b$ tends to infinity.

The parameter $\Delta$ can be re-expressed in terms of $\Delta_{0}(T)$, which is the BCS gap in the absence of impurities, and $b_{0}=2 U_{i}(\pi) / \Delta_{0}(T)$, which linearly depends on the impurity strength. The relation between $\delta=\Delta / \Delta_{0}$ and $b_{0}$ (and between $b=b_{0} / \delta$ and $b_{0}$ ) is obtained from the self-consistent condition on the order parameter

$$
\Delta=u_{e f f} \int_{0}^{\omega_{\max }} \frac{\bar{\Delta}_{\omega_{m}}}{\sqrt{\bar{\Delta}_{\omega_{m}}^{2}+\omega_{m}^{2}}},
$$



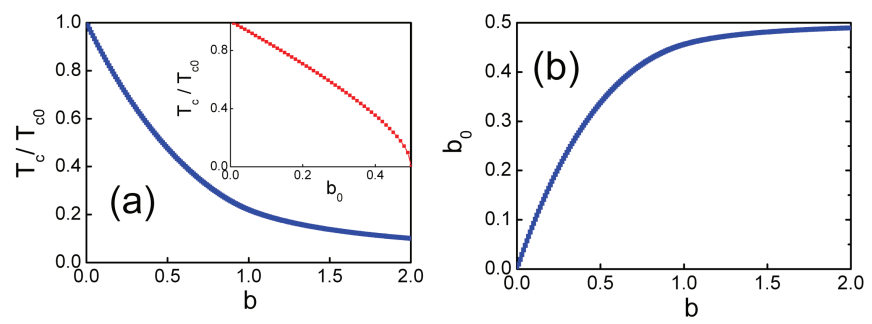

FIG. 7: $\quad \mathrm{T}_{c}$ (a) and $b_{0}=2 U_{i}(\pi) / \Delta_{0}$ (b) as functions of $b=2 U_{i}(\pi) / \Delta$, where $\Delta$ is the order parameter, and $\Delta_{0}$ is the gap in the absence of impurities. The inset in (a) shows the dependence of $T_{c}$ on $b_{0}$.

$$
\Delta_{0}=u_{e f f} \int_{0}^{\omega_{\max }} \frac{\Delta_{0}}{\sqrt{\Delta_{0}^{2}+\omega_{m}^{2}}}
$$

where $u_{e f f}$ is the normalized interaction in the $s^{+}$channel. Solving these equations, we obtain after some algebra the relations which express $b_{0}$ in terms of $b$. They are

$$
\begin{aligned}
& b_{0}=b \exp \left(-\frac{\pi b}{4}\right), b<1 \\
& b_{0}=b \exp \left(\frac{1}{2} \sqrt{1-\frac{1}{b^{2}}}-\frac{b}{2} \sin ^{-1} \frac{1}{b}-\cosh ^{-1} b\right), b>1
\end{aligned}
$$

The first regime corresponds to $\delta=b_{0} / b>b_{0}$, and holds for $b_{0}<e^{-\pi / 4} \approx 0.465$. The second regime corresponds to $b>1$ and describes a gapless superconductivity $\left(\bar{\Delta}_{\omega_{m}=0}=0\right)$. When the order parameter $\Delta$ tends to zero, and $b$ tends to infinity, $b_{0}$ approaches 0.5 . We plot $b_{0}$ vs $b$ in Fig. 7 (b).

The vanishing of superconductivity at $T=0$ when $b_{0}$ approaches $1 / 2$ also follows from the generic dependence of $T_{c}$ on the impurity strength. The calculation parallels the one for an $s$-wave superconductor with magnetic impurities [46] and yields

$$
\ln \frac{T_{c}^{0}}{T_{c}}=\psi\left(\frac{1}{2}+\frac{3.53 b_{0}}{4 \pi} \frac{T_{c}^{0}}{T_{c}}\right)-\psi\left(\frac{1}{2}\right)
$$

where $\psi(x)$ is the diGamma function. One can easily check that $T_{c}$ vanishes when $b_{0}$ approaches $1 / 2$. We plot $T_{c}(b)$ and $T_{c}\left(b_{0}\right)$ in Fig. 团(a).

\section{Spin response of an $s^{+}$superconductor}

The dynamical spin susceptibility of a superconductor is given by an RPA-type formula

$$
\chi_{s}(\mathbf{q}, \Omega)=\frac{\chi_{s}^{0}(\mathbf{q}, \Omega)}{1-\Gamma_{s d w}^{(r)} \chi_{s}^{0}(\mathbf{q}, \Omega)},
$$

where $\chi_{s}^{0}(q, \Omega)$ is the (dimensionless) susceptibility of an ideal $s^{+} \mathrm{SC}$ (the sum of $G G$ and $F F$ terms with spin matrices in the vertices). In our case, when $\epsilon_{\mathbf{k}}^{c} \approx-\epsilon_{\mathbf{k}+\mathbf{Q}}^{f}$, and the gap changes sign between hole and electron FS, one can easily verify that $\chi_{s}^{0}(\mathbf{q} \approx \mathbf{Q}, \Omega)$ coincides with the particle-particle susceptibility for either $c-$ or $f-$ fermions. This leads to several consequences.

1. In the normal state, $\chi_{s}^{0}(\mathbf{Q}, \Omega)=\log E_{F} /(-i \Omega)$, that is $\operatorname{Im}_{s}(\mathbf{Q}, \omega)$ only weakly (logarithmically) depends on frequency. This could be verified in INS experiments.

2. In a superconducting state, $\chi_{s}(\mathbf{Q}, \Omega)$ has a resonance below $2 \Delta$. Indeed, at $T=0$, in the clean limit and at small $\Omega$ and $\mathbf{q}-\mathbf{Q}$,

$$
\chi_{s}^{0}(\mathbf{q}, \Omega)=\log \frac{E_{F}}{E_{0}}+\frac{1}{4 \Delta^{2}}\left(\Omega^{2}-v^{2}(\mathbf{q}-\mathbf{Q})^{2}\right)
$$

where $v=v_{F} / \sqrt{2}$ is the velocity of the AndersonBogolyubov mode in two dimensions (2D), and $E_{0}$ is the largest of $\Delta$ and the cutoff energy associated with non-equivalence of the two FS. Substituting this into $\chi_{s}(q, \Omega)$, and assuming $\Gamma_{s d w}^{(r)} \log E_{F} / E_{0}<$ 1, i.e., no SDW instability, we find the resonance at $\Omega=\sqrt{\left(v^{2}(\mathbf{q}-\mathbf{Q})^{2}+\Omega_{0}^{2}\right.}$, where $\Omega_{0}=2 \Delta\left(1 / \Gamma_{s d w}^{(r)}-\right.$ $\left.\log E_{F} / E_{0}\right)^{1 / 2}$. This resonance has been earlier obtained in the numerical analysis in Refs. [23, 47]. It bears both similarities and differences with the spin resonance in $d_{x^{2}-y^{2}}$ SC. On one hand, both are excitonic resonances, and both occur because the gap changes sign between the FS points $\mathbf{k}$ and $\mathbf{k}+\mathbf{Q}$. On the other hand, the resonance frequency in a $d_{x^{2}-y^{2}} \mathrm{SC}$ disperses downwards because of the nodes, while for a nodeless $s^{+} \mathrm{SC}$, the resonance disperses upwards, with large velocity. Indeed, this is only valid if $\Omega \ll 2 \Delta$, otherwise the dispersion becomes more complex.

Note in passing that, because the two gaps have opposite signs, there should also exist a resonance mode in the particle-particle channel, at momentum $\mathbf{k}=\mathbf{Q}$, similar to the Leggett mode in a twoband superconductor [48].

3. An $s^{+}$superconductor has a rather peculiar lowfrequency behavior of $\operatorname{Im} \chi_{s}(\mathbf{q} \sim \mathbf{Q}, \Omega \rightarrow 0)$. In the clean limit,

$$
\left.\frac{\operatorname{Im} \chi_{s}^{0}(\mathbf{q}, \Omega)}{\Omega}\right|_{\Omega=0} \propto \sum_{\mathbf{k}} C_{\mathbf{k}, \mathbf{q}} \frac{\partial n_{F}\left(E_{\mathbf{k}}\right)}{\partial E_{\mathbf{k}}}
$$

where $E_{\mathbf{k}}=\sqrt{\Delta^{2}+\epsilon_{\mathbf{k}}^{2}}, n_{F}(E)$ is Fermi function, and $C_{\mathbf{k}, \mathbf{q}}=1+\left(\epsilon_{\mathbf{k}}^{c} \epsilon_{\mathbf{k}+\mathbf{q}}^{f}+\Delta^{c} \Delta^{f}\right) /\left(E_{\mathbf{k}}^{c} E_{\mathbf{k}+\mathbf{q}}^{f}\right)$ is the coherence factor. We see that the coherence factor vanishes identically for $\mathbf{q}=\mathbf{Q}$ such that $\operatorname{Im} \chi_{s}^{0}(\mathbf{q}, \Omega) / \Omega_{\mid \Omega=0} \propto(\mathbf{q}-\mathbf{Q})^{2}$. 
NMR spin-lattice relaxation rate

The spin-lattice relaxation rate measured by nuclear magnetic resonance (NMR) is given by

$$
\begin{aligned}
\frac{1}{T_{1}} & \left.\propto T \sum_{\mathbf{q}} \frac{\operatorname{Im} \chi_{s}(\mathbf{q}, \Omega)}{\Omega}\right|_{\Omega=0} \\
& \propto T \sum_{\mathbf{q}} \chi_{s}^{2}(\mathbf{q}, 0)\left[\frac{\operatorname{Im} \chi_{s}^{0}(\mathbf{q}, \Omega)}{\Omega}\right]_{\Omega=0} .
\end{aligned}
$$

Because $\chi_{s}(\mathbf{q}, \Omega=0)$ is enhanced near $\mathbf{Q}$, this region contributes most to the momentum sum. The smallness of $\operatorname{Im} \chi_{s}^{0}(\mathbf{q}, \Omega) / \Omega_{\mid \Omega=0}$ for $\mathbf{q} \sim \mathbf{Q}$ then implies that $1 / T_{1}$ has extra smallness in a clean $s^{+} \mathrm{SC}$ [by the same reason, there is no Hebel-Slichter peak in $1 / T_{1}$ near $T_{c}$ ].

In the presence of impurities, $\operatorname{Im} \chi_{s}^{0}(\mathbf{Q}, \Omega) / \Omega_{\mid \Omega=0}$ remains nonzero, and $1 / T_{1} \approx$ $T \operatorname{Im} \chi_{s}(\mathbf{Q}, \Omega) / \Omega_{\mid \Omega=0} \int d^{2} q \chi_{s}^{2}(\mathbf{q}, \Omega=0)$. The full expression for $1 / T_{1}$ is rather involved as one has to include the full $G$ and $F$, and the full vertex. It simplifies considerably if we neglect vertex corrections and assume that intraband scattering $U_{i}(0)$ (harmless for superconductivity) well exceeds $\Delta$. In this case, we obtained analytically, at a finite $T$,

$$
\begin{aligned}
& \frac{1}{T_{1}}=\left.\frac{1}{T_{1}}\right|_{T_{c}} \times \\
& \quad \int_{0}^{\infty} \frac{d x}{4 \cosh ^{2} \frac{x}{2 T}}\left(1-\frac{|\bar{\Delta}|^{2}-x^{2}}{\sqrt{\left(|\bar{\Delta}|^{2}-x^{2}\right)^{2}+4 x^{2}\left(\bar{\Delta}^{\prime \prime}\right)^{2}}}\right)
\end{aligned}
$$

where $\bar{\Delta}$ is given by Eq. (18) with BCS $T$-dependent $\Delta(T)$. We verified numerically that lowest order vertex corrections do not change the result in any significant way.

In Fig 8 we plot the normalized temperature dependence of $1 / T_{1}(T)$ for several values of $b=2 U_{i}(\pi) / \Delta(T=$ $0)$. Stronger impurity scattering corresponds to larger $b$ [it doesn't make a difference wthether to parametrize the impurity strength in terms of $b$, which depends on impurity strength in a complex way, or $b_{0}$, which scales linearly with the impurity strength, because of one-toone correspondence between $b$ and $b_{0}$, see Eq. (20)].

For $b<1$, the low- $T$ behavior is exponential, as is expected for a superconductor without nodes. However, we see that for $b \geq 0.3$, there is a wide intermediate $T$ range where the behavior of $1 / T_{1}$ closely resembles a powerlaw $T^{\alpha}$. The exponent $\alpha$ decreases as $b$ increases from $\alpha \approx 3$ for $b=0.3$ to $\alpha \approx 2$ for $b=0.9$. The $T^{3}$ behavior was suggested based on experimental fits and was presented as evidence for $d$-wave superconductivity in $\mathrm{Fe}$ pnictides. Our results show that $1 / T_{1}(T)$ in a dirty $s^{+}$ superconductor mimics a power-law over a wide $T$ range even when the DOS still vanishes at $\omega=0$, and $T_{c}$ is only slightly affected by impurities. Furthermore, we argue,
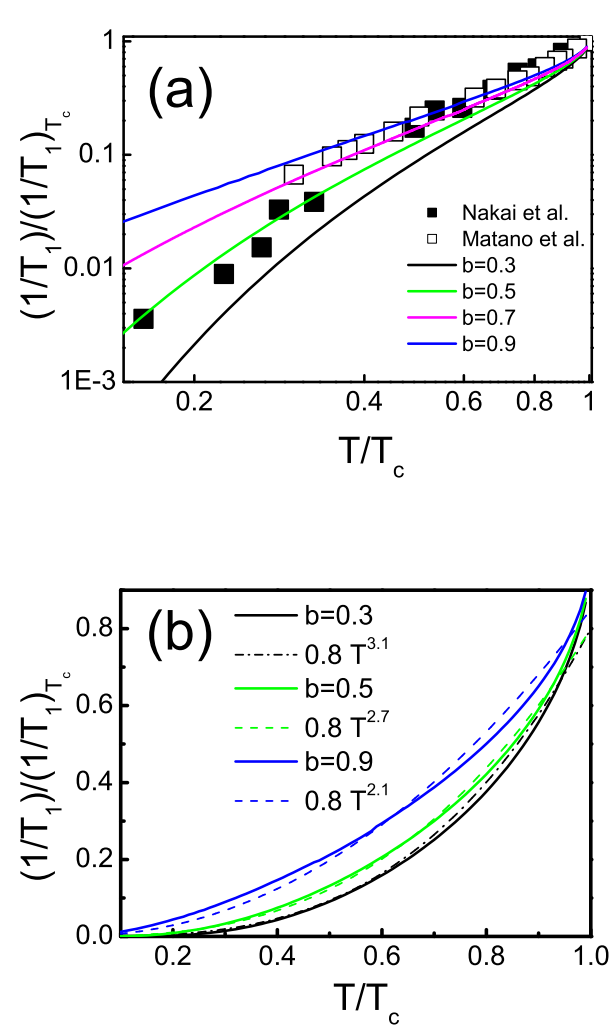

FIG. 8: (color online) (a) Calculated temperature dependence of $1 / T_{1}$ for an $s^{+}$superconductor with nonmagnetic impurities. The normalization is chosen such that $1 / T_{1}=1$ at $T=T_{c}$. The theoretical curves are for various values of the parameter $b=2 U_{i}(\pi) / \Delta(T=0)$ which measures the strength of the pair-breaking component of non-magnetic impurities. Gapless superconductivity occurs for $b>1$. The experimental data are taken from Refs. $\left[32, \underline{34}\right.$. (b) Theoretical $1 / T_{1}$ for different $b$ vs power-law forms . All theoretical dependencies are exponential in $T$ at very low $T$, but are described by power-laws $T^{\alpha}$ over a wide $T$ range below $T_{c}$. The exponent $\alpha$ decreases as $b$ increases from $\alpha \approx 3$ for $b=0.3$ to $\alpha \approx 2$ for $b=0.9$.

based on Fig. 8(a) that the experimental $T$ dependence of $1 / T_{1}$ can only approximately be fitted by a particular power of $T$. We believe that the reported power-law form reflects intermediate asymptotics of a complex $T$ behavior of $1 / T_{1}$, and one should reduce temperature further to be able to distinguish between a true power-law and exponential behavior [49].

Note in passing that the theoretical behavior is exponential at the lowest $T$ only if $b<1$. For $b=1$, which is the critical $b$ for a gapless $s^{+} \mathrm{SC}, 1 / T_{1} \propto T^{5 / 3}$ at the lowest $T$, and for larger $b, 1 / T_{1}(T) \propto T$. 


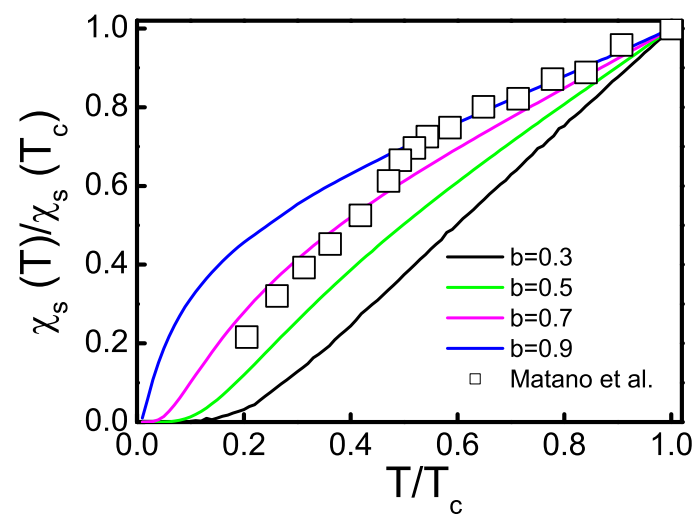

FIG. 9: (color online) Calculated temperature dependence of the uniform susceptibility for various values of $b$. The experimental data are taken from Ref. 34].
Uniform susceptibility

Finally, we also computed uniform spin susceptibility $\chi_{s}(T) \approx \chi_{s}^{0}(q=\Omega=0)$, measured by Knight shift. It is obtained by standard means [50], and for a superconductor with $s^{+}$gap symmetry is given by

$$
\begin{aligned}
\chi_{s}(T) & =\chi_{s}\left(T_{c}\right)\left[1-\int_{0}^{\infty} \tanh \frac{x}{2 T} \operatorname{Im} \frac{\bar{\Delta}^{2}}{\left(\bar{\Delta}^{2}-x^{2}\right)^{3 / 2}-2 U_{i}(\pi) x^{2}}\right] \\
& =\chi_{s}\left(T_{c}\right)\left[1-\int_{0}^{\infty} \tanh \frac{x}{2 T} \frac{1}{\Delta(T)} \operatorname{Im} \frac{1}{\left(1-u_{x}^{2}\right)^{3 / 2}-b_{T}}\right]
\end{aligned}
$$

where $u_{x}=x / \bar{\Delta}$ and $\bar{\Delta}$ depends on $x$ and is given by (18)

We emphasize that ladder series of vertex corrections must be included in the calculation of $\chi_{s}(T)$ to recover $S U(2)$ spin symmetry. Observe that $U_{i}(0)$ drops from the expression for $\chi_{s}(T)$, because impurity scattering of electron within either hole or electron FS does not differentiate between a conventional $s$-wave and $s^{+}$gap symmetry.

Eq. (27) is similar, but not identical to the expression for $\chi_{s}(T)$ in an ordinary s-wave superconductor with magnetic impurities [50]. In both cases, $\chi_{s}(T)$ differs from free-fermion value. However, for magnetic impurities, $\chi_{s}(T=0)$ becomes finite for any nonzero strength of the impurity scattering, while in our case, the impurities are actually non-magnetic, and $\chi_{s}(T)$ still vanishes at $T=0$ for all $b<1$, for which the DOS still vanishes at zero frequency.

We plot Eq. (27) in Fig. 9 for the same $b$ as $1 / T_{1}$. We see the same trend as in Fig. 8 the theoretical $T$ dependence of $\chi_{s}(T)$ is exponential in $\Delta / T$ at the lowest $T$, but rapidly deviates from exponent already at small $T$, and is roughly a power-law in $T$ in the same $T$ range where $1 / T_{1}(T)$ can be fitted by a power-law. This is another indication that one should perform Knight shift and $1 / T_{1}$ measurements down very low $T$ to be able to distinguish between the nodeless $s^{+}$state and a SC state with gap nodes. Note also that the same $b=0.7$ which fits $1 / T_{1}$ data by Matano et al 34] also fits reasonably well their data on the Knight shift.

\section{CONCLUSIONS}

To conclude, in this paper we presented Fermi liquid analysis of SDW magnetism and superconductivity in $F e$-pnictides. We considered a two-band model with small hole and electron pockets located near $(0,0)$ and $\mathbf{Q}=(\pi, \pi)$ in the folded BZ. We argued that for such geometry, particle-hole and particle-particle channels are nearly identical, and the interactions logarithmically increase at low energies. We found that the interactions in the SDW and extended $s$-wave channels $\left(\Delta_{\mathbf{k}}=-\Delta_{\mathbf{k}+\mathbf{Q}}\right)$ become comparable in strength due to the increase of the intraband pair hopping term and the reduction of the Hubbard-type intraband repulsive interaction. We argued that at zero doping, SDW instability comes first, but at a finite doping, $s^{+}$superconducting instability occurs at a higher $T$.

This $s^{+}$pairing bears similarity to magnetically mediated $d_{x^{2}-y^{2}}$ pairing in systems with large FS with hot spots in the sense that in both cases the pairing comes from repulsive interaction, peaked at $\mathbf{Q}$, and requires the gap to change its sign under $\mathbf{k} \rightarrow \mathbf{k}+\mathbf{Q}$. the difference is that for small pockets, the gap changes sign away from the FS and remains constant along the FS.

We analyzed spin response of a clean and dirty $s^{+}$ superconductor and found that (i) it possess a resonance mode which disperses with the same velocity as Anderson-Bogolyubov mode, (ii) intraband scattering by non-magnetic impurities is harmless, but interband scat- 
tering affects the system in the same way as magnetic impurities in an $s$-wave SC, (iii) $1 / T_{1}$ has an extra smallness in the clean limit due to vanishing of the coherence factor, (iv) in the presence of impurities, there exists a wide range of $T$ where the $T$-dependencies of $1 / T_{1}$ and the uniform susceptibility for an $s^{+} \mathrm{SC}$ resemble the ones for a SC with nodes.

Note added While completing this work we became aware that similar results for spin-lattice relaxation rate, $1 / T_{1}$, in the superconducting state have been obtained in Ref. 51].

We acknowledge helpful discussions with Ar. Abanov, J. Betouras, O. Dolgov, K. Ishida, M. Korshunov, I Mazin, J. Schmalian, Z. Tesanovic, and G.-q. Zheng. AVC has been supported by NSF-DMR 0604406 and MPI for Physics of Complex Systems in Dresden.

[1] Y. Kamihara, T. Watanabe, M. Hirano, and H. Hosono, J. Am. Chem. Soc. 1303296 (2008).

[2] X.H. Chen, T. Wu, G. Wu, R.H. Liu, H. Chen, and D.F. Fang, Nature 453761 (2008).

[3] G.F. Chen, Li Z., Wu D., Li G., Hu W.Z., Dong J., Zheng P., Luo J.L., Wang N.L. Phys. Rev. Lett. 100247002 (2008).

[4] Z.-A. Ren, Yang J., Lu W., Yi W., Che G.-C., Dong X.L., Sun L.-L., Zhao Z.-X. arXiv:0803.4283 (unpublished).

[5] M. Rotter, M. Tegel and D. Johrendt, arXiv:condmat/0805.4630 (2008).

[6] J. Dong, H. J. Zhang, G. Xu, Z. Li, G. Li, W. Z. Hu, D. Wu, G. F. Chen, X. Dai, J. L. Luo, Z. Fang, N. L. Wang, Europhys. Lett. 83, 27006 (2008).

[7] Clarina de la Cruz, Q. Huang, J. W. Lynn, J. Li, W. Ratcliff II, J.L. Zarestky, H.A. Mook, G.F. Chen, J.L. Luo, N.L. Wang, and P. Dai, Nature 453, 899 (2008).

[8] T. Nomura, S.W. Kim, Y. Kamihara, M. Hirano, P.V. Sushko, K. Kato, M. Takata, A.L. Shluger, and H. Hosono, arXiv:0804.3569 (unpublished).

[9] H.-H. Klauss, H. Luetkens, R. Klingeler, C. Hess, F.J. Litterst, M. Kraken, M. M. Korshunov, I. Eremin, S.-L. Drechsler, R. Khasanov, A. Amato, J. Hamann-Borreo, N. Leps, A. Kondrat, G. Behr, J. Werner, B. Büchner, Phys. Rev. Lett. 101, 077005 (2008).

[10] Qimiao Si, and Elihu Abrahams, Phys. Rev. Lett. 101, 076401 (2008).

[11] M. Daghofer, A. Moreo, J.A. Riera, E. Arrigoni, D.J. Scalapino, E. Dagotto, arXiv:0805.0148 (unpublished).

[12] F. Ma, and Z.-Y. Lu, Phys. Rev. B 78, 033111 (2008).

[13] S. Raghu, X.-L. Qi, C.-X. Liu, D. Scalapino, and S.-C. Zhang, Phys. Rev. B 77 220503(R) (2008).

[14] C. Cao, P.J. Hirschfeld, H.-P. Cheng, Phys. Rev. B 77, 220506(R) (2008).

[15] S. Lebègue, Phys. Rev. B 75, 035110 (2007).

[16] D. Singh and M.-H. Du, Phys. Rev. Lett. 100, 237003 (2008).

[17] L. Boeri, O.V. Dolgov, and A.A. Golubov, Phys. Rev. Lett. 101, 026403 (2008).

[18] I.I. Mazin, D.J. Singh, M.D. Johannes, and M.H. Du, Phys. Rev. Lett. 101, 057003 (2008).
[19] K. Kuroki, S. Onari, R. Arita, H. Usui, Y. Tanaka, H. Kontani, and H. Aoki, Phys. Rev. Lett. 101, 087004 (2008).

[20] C. Liu, G.D. Samolyuk, Y. Lee, N. Ni, T. Kondo, A.F. Santander-Syro, S.L. Bud'ko, J.L. McChesney, E. Rotenberg, T. Valla, A. V. Fedorov, P.C. Canfield, B.N. Harmon, A. Kaminski, arXiv:0806.3453 (unpublished); C. Liu, T. Kondo, M.E. Tillman, R. Gordon, G.D. Samolyuk, Y. Lee, C. Martin, J.L. McChesney, S. Bud'ko, M.A. Tanatar, E. Rotenberg, P.C. Canfield, R. Prozorov, B.N. Harmon, and A. Kaminski, arXiv:0806.2147 (unpublished);

[21] L.X. Yang, Y. Zhang, H.W. Ou, J.F. Zhao, D.W. Shen, B. Zhou, J. Wei, F. Chen, M. Xu, C. He, Y. Chen, Z.D. Wang, X.F. Wang, T. Wu, G. Wu, X.H. Chen, M. Arita, K. Shimada, M. Taniguchi, Z.Y. Lu, T. Xiang, D.L. Feng, arXiv:0806.2627 (unpublished).

[22] V. Barzykin and L.P. Gorkov, JETP Lett. 88, 142 (2008).

[23] M.M. Korshunov, and I. Eremin, arXiv:0804.1793 (unpublished).

[24] V. Cvetkovic and Z. Tesanovic, arXiv:0804.4678 (unpublished).

[25] F. Wang, H. Zhai, Y. Ran, A. Vishwanath, and D.-H. Lee, arXiv:0805.3343 (unpublished)

[26] F. Wang, H. Zhai, Y. Ran, A. Vishwanath, and D.-H. Lee, arXiv:0807.0498 (unpublished)

[27] J. Lorenzana, G. Seibold, C. Ortix, and M. Grilli, arXiv:0807.2412v1 (unpublished).

[28] T. Kondo, A.F. Santander-Syro, O. Copie, C. Liu, M.E. Tillman, E.D. Mun, J. Schmalian, S.L. Bud'ko, M.A. Tanatar, P.C. Canfield, and A. Kaminski, arXiv:0807.0815 (unpublished).

[29] H. Ding, P. Richard, K. Nakayama, T. Sugawara, T. Arakane, Y. Sekiba, A. Takayama, S. Souma, T. Sato, T. Takahashi, Z. Wang, X. Dai, Z. Fang, G.F. Chen, J.L. Luo, and N.L. Wang, Europhys. Lett. 83, 47001 (2008).

[30] T. Kondo, A.F. Santander-Syro, O. Copie, C. Liu, M.E. Tillman, E.D. Mun, J. Schmalian, S.L. Bud'ko, M.A. Tanatar, P.C. Canfield, and A. Kaminski, arXiv:0807.0815 (unpublished).

[31] T. Y. Chen, Z. Tesanovic, R. H. Liu, X. H. Chen, C. L. Chien, Nature 453, 1224 (2008).

[32] Y. Nakai, K. Ishida, Y. Kamihara, M. Hirano, and H. Hosono J. Phys. Soc. Jpn. 77, 073701 (2008).

[33] H.-J. Grafe, D. Paar, G. Lang, N.J. Curro, G. Behr, J. Werner, J. Hamann-Borrero, C. Hess, N. Leps, R. Klingeler, and B. Buechner, Phys. Rev. Lett. 101, 047003 (2008).

[34] K. Matano, Z.A. Ren, X.L. Dong, L.L. Sun, Z.X. Zhao, and G.-q. Zheng, Europhys. Lett. 83, 57001 (2008).

[35] Cenke Xu, Markus Mueller, and Subir Sachdev, Phys. Rev. B 78, 020501(R) (2008); Cenke Xu, Yang Qi, and Subir Sachdev, arXiv:0807.1542.

[36] Note that our two-band model differs conceptually from the two-band model based on the $d_{y z}$ and $d_{x z}$ orbitals considered in [13]. Band structure calculations [17] show that not only $d_{y z}$ and $d_{x z}$ orbitals, but all Fe $5 d$-orbitals contribute to the bands crossing the Fermi level, and, besides, Fe $5 d$-orbitals strongly hybridize with the As $p$-states. The tight-binding model of the pnictides then should include 8 bands in the unfolded BZ. Our model with two hybridized orbitals and two bands crossing the Fermi level is not related to actual Fe $5 d$ and As $p$-orbitals, and is simply a phenomenological minimal 
model to describe SDW and superconductivity.

[37] J. Wu, P. Phillips, and A.H. Castro-Neto, arXiv:0805.2167.

[38] A. A. Abrikosov, L. P. Gorkov, and I. E. Dzyaloshinski, Methods of quantum field theory in statistical physics, (Dover Publications, New York, 1963); E. M. Lifshitz and L. P. Pitaevski, Statistical Physics, (Pergamon Press, 1980).

[39] A.T. Zheleznyak, V.M. Yakovenko, and I.E. Dzyaloshinskii, Phys. Rev. B 55, 3200 (1997).

[40] W.L. McMillan, Phys. Rev. 167, 331 (1968).

[41] E. Shender, Sov. Phys. JETP, 56, 178 (1982); A. Pimpinelli, E. Rastelli, and A. Tassi, J. Phys.: Cond. Matt 1, 2131 (1989); A. Moreo et al., Phys. Rev. B 42, 6283 (1990); A. Chubukov, Phys. Rev. B 44, 392 (1991).

[42] P. Chandra, P. Coleman, and A.I. Larkin, Phys. Rev. Lett. 64, 88 (1990).

[43] For recent literature see e.g., T. Dahm, P. J. Hirschfeld, D. J. Scalapino, and L. Zhu, Phys. Rev. B 72, 214512
(2005).

[44] A.A. Golubov, and I.I. Mazin, Phys. Rev. B 55, 3200 (1997).

[45] G. Preosti, and P Muzikar, Phys. Rev. B 54, 3489 (1996).

[46] A.A. Abrikosov and L.P. Gorkov, Sov. Phys. JETP 12, 1243 (1961).

[47] T.A. Maier, and D.J. Scalapino, Phys. Rev. B 78, 020514(R) (2008).

[48] A.J. Leggett, Prog. Theor. Phys. 36, 901 (1966).

[49] The behavior of $1 / T_{1}$ is further complicated by the presence of four Fermi surfaces and the variation of the gap size between the two Fermi surfaces near the same momenta, as evidenced by ARPES measurements 29].

[50] L.P. Gorkov and A.I. Rusinov, Sov. Phys. JETP, 19, 922 (1964); W. A. Roshen and J. Ruvalds, Phys. Rev. B 31, 2929 (1985).

[51] D. Parker, O. Dolgov, M.M. Korshunov, A. Golubov, and I.I. Mazin, arXiv:0807.3729 (unpublished). 